\title{
Comparison of Pain Characteristics in Patients with Rheumatoid Arthritis and Systemic Sclerosis with Particular Reference to the Neuropathic Pain Component: Cross-Sectional Study
}

\author{
Gizem Cengiz $^{\mathrm{a}}$ Kemal Erol $^{\mathrm{a}}$ Kevser Gok ${ }^{\mathrm{b}}$ Salih Ozgocmen ${ }^{\mathrm{c}}$ \\ ${ }^{a}$ Division of Rheumatology, Department of Physical Medicine and Rehabilitation, ERU Gevher Nesibe Hospital,

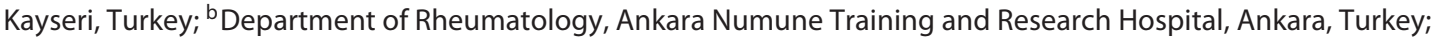 \\ 'Department of Rheumatology, Istinye University Medical Park Gaziosmanpasa Hospital, Istanbul, Turkey
}

\section{Significance of the Study}

- We compared characteristics of neuropathic pain (NeP) in patients with rheumatoid arthritis (RA) and systemic sclerosis (SSc). The NeP component was similar in the two conditions; however, NeP was associated with a heavier burden of disease in patients with RA. Coexisting $\mathrm{NeP}$ should be carefully evaluated in patients with RA and SSc.

\section{Keywords}

Pain · Neuropathic pain · PainDetect questionnaire · Rheumatoid arthritis · Systemic sclerosis

\begin{abstract}
Objective: The aim of the study was to compare characteristics of pain in terms of neuropathic pain (NeP) and to assess the association between the neuropathic component and quality of life (QoL) in patients with systemic sclerosis (SSc) and rheumatoid arthritis (RA). Subjects and Methods: Fiftyfour patients (47 females, 7 males) with SSc and 53 patients (46 females, 7 males) with RA were assessed for outcome measures including disease activity, physical functions, mental condition and health-related QoL (HRQoL) measures
\end{abstract}

(Short Form-36; Hospital Anxiety and Depression Scale), and pain. NeP was assessed by the Douleur Neuropathique 4 (DN4) and PainDetect questionnaires in this cross-sectional study. Results: The patients had similar education, smoking status, functioning, and HRQoL. However, the patients with RA declared a more severe visual analogue scale of pain and a higher BMI than those with SSc. The NeP component was detected in $42.6 \%(n=23)$ of the SSc patients and in $45.3 \%$ ( $n=24)$ of the RA patients $(p>0.05)$ according to DN4. On PainDetect, possible NeP was detected in $13.0 \%(n=7)$ versus $15.1 \%(n=8)$, whereas $16.7 \%(n=9)$ versus $17.0 \%(n=9)$ were likely to have NeP in SSc and RA, respectively ( $p>0.05)$. Most of the NeP characteristics were similar in SSc and RA, except for numbness and painful cold, which were notably more common in patients with SSc. Having the NeP compo-

\begin{tabular}{ll}
\hline KARGER & $\begin{array}{l}\text { (c) } 2018 \text { The Author(s) } \\
\text { Published by S. Karger AG, Basel }\end{array}$ \\
E-Mail karger@karger.com & $\begin{array}{l}\text { This is an Open Access article licensed under the Creative Commons } \\
\text { Attribution-NonCommercial-4.0 International License (CC BY-NC) } \\
\text { (http://www.karger.com/Services/OpenAccessLicense), applicable to } \\
\text { the online version of the article only. Usage and distribution for } \\
\text { commercial purposes requires written permission. }\end{array}$
\end{tabular}


nent (according to DN4) had no influence on functioning and HRQoL in SSc; however, the NeP component revealed a heavier burden of disease regarding functional status, HRQoL, and psychometric components in RA. Conclusion: The NeP component was similar between patients with SSC and RA. However, NeP was associated with a heavier burden of disease in patients with RA. @ 2018 The Author(s)

Published by S. Karger AG, Basel

\section{Introduction}

Rheumatoid arthritis (RA) is a systemic inflammatory disease characterized by progressive, persisting synovitis and structural damages leading to severe disability with functional loss [1]. Despite evolving therapies in the field of RA, some patients still suffer from a high level of persistent pain. A comprehensive assessment including the whole aspect of pain in patients with RA could take place with more advanced pain management protocols and good patient outcomes [2].

Systemic sclerosis (SSc) is an autoimmune disease related to increasing fibrosis of the skin and some internal organs [3-5]. Only a few studies have investigated pain and its impact on health-related quality of life (HRQoL) and other parameters of disease-related outcomes and $\operatorname{mood}[6-8]$.

Pain is usually an important complaint in most patients with rheumatic diseases $[9,10]$. Neuropathic pain $(\mathrm{NeP})$ in rheumatic diseases is common and is associated with a lesser QoL and a heavier burden of disease $[11,12]$. Furthermore, studies assessing the neuropathic component of pain in the general population are scanty and report a predicted prevalence changing from 7 to $10 \%[13$, 14]. Pain in RA may be of nociceptive and non-nociceptive origins, which lead to different pain characteristics detected by various tools $[8,10]$. Despite clinical remission in RA, some patients may continue to declare unexpectedly higher levels of pain [15-17]. The International Association for the Study of Pain (IASP) has introduced the concept of $\mathrm{NeP}$, defined as pain initiated or caused by a lesion or disease in the somatosensory system. Recently, experts have defined $\mathrm{NeP}$ as a direct consequence of a lesion or disease related to the somatosensory system [18].

In this study, we aimed to compare pain characteristics particularly in terms of $\mathrm{NeP}$ and to evaluate the possible impact of the neuropathic component on QoL in patients with RA and SSc.

\section{Subjects and Methods}

\section{Participants}

Fifty-four patients (47 females, 7 males) with SSc and 53 patients (46 females, 7 males) with RA were included. For this crosssectional study, patients who met the 2013 American College of Rheumatology (ACR)/European League against Rheumatism (EULAR) collaborative criteria for SSc and the 2010 American College of Rheumatology (ACR)/European League against Rheumatism (EULAR) collaborative criteria for RA were recruited [19, 20]. Patients with a prior diagnosis or those taking medications for $\mathrm{NeP}$, mood disorders (including antidepressants, antipsychotics, and antiepileptics or continuous analgesics), uncontrolled diabetes or neurological disorders, or taking any biologic agents for their treatments were excluded.

\section{Procedures and Measures}

All subjects were evaluated for disease-specific and generic outcome measures including disease activity parameters, physical functions, mental status, and HRQoL measures (Short Form36; Health Assessment Questionnaire; Hospital Anxiety and Depression Scale). Symptom duration was defined as the beginning of Raynaud's phenomenon in SSc and arthritis in RA. The 6-min walking distance was assessed by the 6 -min walking test noted in meters [21]. The visual analogue scale of pain (0-10 scale) within the last week was noted [22]. NeP assessed by the Douleur Neuropathique 4 (DN4) interview [23] and the PainDetect questionnaire were applied by the same experienced physician who was blinded to the clinical findings of the patient and outcome data.

The DN4 questionnaire consisted of 10 items. The first 7 items were related to self-reported pain sensation including burning, tingling, pins and needles, painful cold, electric shock, numbness, and itching. Three items (hypoesthesia to touch, hypoesthesia to prick, and brushing) were related to clinical findings. Patients with a score $\geq 4$ in DN4 were considered as having "probable NeP." In both groups, all patients had pain complaints in the upper extremities; therefore, DN4 was applied to the upper extremities, hand and wrist joints, and skin between the hands and elbows.

The PainDetect questionnaire is a NeP screening tool consisting of 3 parts and 12 items. The first 7 items detect the pain gradation with each item scored from 0 to 5 points; the other 4 items are related to the pattern of pain course, and 1 item is related to radiating pain. The score of the PainDetect questionnaire ranged from 1 to 38 . Patients with a score of $0-12$ were considered negative. $\mathrm{Pa}$ tients with a score of $13-18$ and $\geq 19$ were considered "possible" and "likely" $\mathrm{NeP}$ patients, respectively [24]. Valid and reliable Turkish versions of the DN4 and PainDetect questionnaires have been published, and we used these versions $[25,26]$. NeP assessment was made by the same physician (G.C.) who was blinded to the patients' clinical data. To prevent bias, physical examinations and the clinical assessments including the health assessments and QoL questionnaires were performed by another physician (K.E.) in the outpatient clinic of ERU Gevher Nesibe Hospital. The study protocol was approved by the local ethics committee of our institution, and written informed consent according to the Declaration of Helsinki was obtained from all patients. 
Table 1. Demographics, disease-specific, and generic outcome measures including physical functions, psychological status, and health-related quality of life (SF-36, HAQ) in the groups

\begin{tabular}{lcccc}
\hline & SSc $(n=54)$ & RA $(n=53)$ & $p$ & $t$ \\
\hline Age, years & $46.65 \pm 13.75$ & $51.19 \pm 10.12$ & 0.054 & -1.92 \\
BMI & $26.54 \pm 6.11$ & $30.95 \pm 6.16$ & 0.001 & -3.57 \\
Symptom duration, years & $9.39 \pm 9.06$ & $10.96 \pm 7.94$ & 0.342 & -0.95 \\
HADS-depression & $6.87 \pm 3.70$ & $7.26 \pm 4.58$ & 0.094 & -0.49 \\
HADS-anxiety & $7.00 \pm 4.67$ & $7.62 \pm 4.59$ & 0.946 & -0.69 \\
VAS-pain & $3.66 \pm 2.96$ & $5.16 \pm 2.09$ & 0.003 & -3.02 \\
6MWT, m & $424.40 \pm 74.86$ & $373.77 \pm 83.60$ & 0.002 & 3.23 \\
HAQ & $0.57 \pm 0.65$ & $0.51 \pm 0.62$ & 0.639 & 0.47 \\
SF-36-PCS & $53.41 \pm 21.70$ & $50.71 \pm 21.11$ & 0.515 & 0.65 \\
SF-36-MCS & $59.24 \pm 21.82$ & $56.39 \pm 22.37$ & 0.506 & 0.66 \\
VAS-fatigue (1-10) & $5.96 \pm 2.73$ & $5.19 \pm 2.79$ & 0.162 & 1.40 \\
\hline
\end{tabular}

VAS, visual analogue scale; 6MWT, 6-min walking test; HAQ, health assessment questionnaire; SF-36-PCS, short-form 36 physical component score; SF-36-MCS, short-form 36 mental component score, HADS, hospital anxiety and depression score.

Statistical Analyses

All data were analyzed by the Statistical Package for Social Sciences (SPSS 20.0; IBM, Armonk, NY, USA). The data of patients with SSc and RA were assessed for normal distribution using the Kolmogorov-Smirnov test. Demographic variables and clinical parameters of patients with RA and SSc were compared using the $t$ test or $\chi^{2}$ test. Patients with DN4 values $<4$ or $\geq 4$ were grouped as $\mathrm{NeP}$ negative and $\mathrm{NeP}$ positive and were compared within each group of RA and SSc using the $t$ test. A $p$ value of $<0.05$ was considered statistically significant.

\section{Results}

Sixty patients with SSc and 60 patients with RA were consecutively recruited. Six patients were excluded because of current use of pregabalin and/or antidepressants with a previous diagnosis of $\mathrm{NeP}$ and mood disorders in the SSc group, and 7 patients in the RA group. Fifty-four patients in the SSc and 53 patients in the RA group consented and completed the study.

\section{Clinical and Demographic Characteristics}

All patients had similar age, gender, education, marital status, work status, and smoking status as well as functioning and HRQoL measures (Table 1). However, patients with RA declared more severe pain on the visual analogue scale of pain $(p=0.003)$ and had a higher BMI than patients with SSc $(p=0.001)$. Thirteen patients had pulmonary involvement documented with respiratory functional tests, diffusion X-ray, and pulmonary arterial pressure measurements.

Pain in Rheumatoid Arthritis and Systemic Sclerosis

\section{NeP Component}

The neuropathic component was similar in patients with SSc versus RA. The neuropathic component was detected in $42.6 \%(n=23)$ of the patients with SSc and in $45.3 \%(n=24)$ of the patients with RA $(p>0.05)$ according to DN4 scores. According to the PainDetect questionnaire, a possible neuropathic component was detected in $13.0 \%(n=7)$ versus $15.1 \%(n=8)$, whereas $16.7 \%(n=9)$ versus $17.0 \%(n=9)$ were likely to have $\mathrm{NeP}$ in SSc and RA, respectively $(p>0.05)$. According to the DN4 questionnaire neuropathic characteristics of pain, defined as burning, electric shock, tingling, pins and needles, and itching, were similar in SSc and RA, except for painful cold and numbness, which were significantly more prevalent in patients with SSc $(50.0 \%, n=27$ vs. $18.9 \%, n=10$, $p=0.001$ and $51.9 \%, n=28$ vs. $24.5 \%, n=13, p=0.004$, respectively).

\section{$\mathrm{NeP}$ and Disease Burden}

In patients with SSc with or without the NeP component (according to DN4) had similar functioning and health-related quality measures (Table 2 ). However, having the $\mathrm{NeP}$ component according to DN4 revealed a heavier burden of disease related to functioning, HRQoL, and psychometric components in patients with RA (Table 3). 
Table 2. Comparison of NeP component +ve and -ve patients (according to DN4) with SSc

\begin{tabular}{lcccc}
\hline & $\begin{array}{c}\text { NeP+ve } \\
(n=23)\end{array}$ & $\begin{array}{l}\text { NeP-ve } \\
(n=31)\end{array}$ & $p$ & $t$ \\
\hline Age, years & $51.78 \pm 11.05$ & $42.84 \pm 14.48$ & 0.013 & -2.47 \\
BMI & $28.08 \pm 6.88$ & $25.43 \pm 5.34$ & 0.149 & -1.47 \\
VAS-pain & $4.29 \pm 3.19$ & $3.19 \pm 2.74$ & 0.192 & -1.32 \\
Symptom duration, years & $10.07 \pm 10.67$ & $8.89 \pm 7.81$ & 0.658 & -0.44 \\
VAS-fatigue & $6.09 \pm 2.39$ & $5.85 \pm 3.02$ & 0.758 & -0.31 \\
HAQ & $0.76 \pm 0.76$ & $0.42 \pm 0.51$ & 0.078 & -1.81 \\
SF-36-PCS & $50.65 \pm 23.14$ & $55.46 \pm 20.72$ & 0.434 & 0.79 \\
SF-36-MCS & $58.10 \pm 24.21$ & $59.42 \pm 20.28$ & 0.946 & 0.07 \\
HADS-depression & $7.78 \pm 4.10$ & $6.19 \pm 3.29$ & 0.134 & -1.53 \\
HADS-anxiety & $7.83 \pm 5.37$ & $6.39 \pm 4.07$ & 0.288 & -1.08 \\
6MWT, m & $410.00 \pm 66.56$ & $434.83 \pm 79.85$ & 0.238 & 1.19 \\
\hline
\end{tabular}

VAS, visual analogue scale; 6MWT, 6-min walking test; HAQ, health assessment questionnaire; SF-36-PCS, short-form 36 physical component score; SF-36-MCS, short-form 36 mental component score, HADS, hospital anxiety and depression score; +/-ve, patients who have (+ve) or do not have (-ve) neuropathic pain component.

Table 3. Comparison of $\mathrm{NeP}$ component +ve and -ve patients (according to DN4) with RA

\begin{tabular}{lccrr}
\hline & $\begin{array}{l}\text { NeP+ve } \\
(n=24)\end{array}$ & $\begin{array}{l}\text { NeP-ve } \\
(n=29)\end{array}$ & $p$ & $t$ \\
\hline Age, years & $50.21 \pm 9.17$ & $52.00 \pm 10.94$ & 0.520 & 0.65 \\
BMI & $31.48 \pm 6.89$ & $30.52 \pm 5.60$ & 0.600 & -0.53 \\
DAS28-CRP & $4.27 \pm 1.08$ & $3.71 \pm 1.05$ & 0.069 & -1.86 \\
VAS-pain & $5.83 \pm 1.74$ & $4.60 \pm 2.23$ & 0.029 & -2.25 \\
Symptom duration, years & $13.35 \pm 9.70$ & $8.98 \pm 5.56$ & 0.058 & -1.96 \\
VAS-fatigue & $6.33 \pm 2.49$ & $4.24 \pm 2.71$ & 0.005 & -2.92 \\
HAQ & $0.48 \pm 0.62$ & $0.53 \pm 0.64$ & 0.772 & 0.29 \\
SF-36-PCS & $40.55 \pm 17.63$ & $59.12 \pm 20.27$ & 0.001 & 3.57 \\
SF-36-MCS & $42.83 \pm 15.06$ & $67.62 \pm 21.33$ & $<0.001$ & 4.94 \\
HADS-depression & $9.29 \pm 4.30$ & $5.59 \pm 4.17$ & 0.003 & -3.17 \\
HADS-anxiety & $10.08 \pm 3.78$ & $5.59 \pm 4.24$ & $<0.001$ & -4.08 \\
6MWT, m & $378.33 \pm 77.27$ & $370.00 \pm 89.68$ & 0.718 & -0.36 \\
\hline
\end{tabular}

VAS, visual analogue scale; 6MWT, 6-min walking test; HAQ, health assessment questionnaire; SF-36-PCS, short-form 36 physical component score; SF-36-MCS, short-form 36 mental component score, HADS, hospital anxiety and depression score; DAS28-CRP, disease activity score 28 joints, C-reactive protein.

\section{Discussion}

Our study revealed that patients with RA and SSc had the $\mathrm{NeP}$ component and that it was associated with a heavier burden of disease in individuals with RA compared to those with SSc. In this study, we assessed the NeP component in RA and SSc by 2 widely used NeP screening tools, the DN4 and PainDetect questionnaires [27, 28], and focused on the $\mathrm{NeP}$ component, which are the main differences from the previous study by Perrot et al. [11].
The NeP component is important in the management of patients with SSc and RA. Only 1 study has previously assessed the $\mathrm{NeP}$ component in RA and SSc using the DN4 and the McGill pain questionnaires, which showed that the pain frequency scores were similar between patients with SSc and RA; the pain dimension scores did not correlate with the disease activity scores in patients with SSc and were remarkably lower than in patients with RA. In accordance with our results, Perrot et al. [11] showed that pain was more frequently mild and less severe in pa- 
tients with SSc than RA. The same study showed a relationship between the $\mathrm{NeP}$ component, higher pain scores, and more frequent catastrophic pain in both diseases.

Koop et al. [12] showed that $17.0 \%$ of the patients with RA were classified as having likely $\mathrm{NeP}$ and $21.4 \%$ as having possible $\mathrm{NeP}$ according to the PainDetect questionnaire; neuropathic-like pain symptoms were independently associated with worse self-reported physical and mental health. Rifbjerg-Madsen et al. [29] reported that non-nociceptive pain was very common in RA and was associated with higher scores of disease activity. A recently published study that assessed the $\mathrm{NeP}$ component in RA by the PainDetect questionnaire showed that medium and higher scores of $\mathrm{NeP}$ had poorer effects on depression, anxiety, fatigue, pain, and the mental component of HRQoL. These patients also had higher disease activity scores independently from inflammatory origins but related to the non-nociceptive pain [30]. On the other hand, higher levels of pain were reported to be unrelated to disease activity as assessed by Disease Activity Score 28 [31].

Chronic diseases such as RA and SSc can negatively influence patients in various ways related to physical and emotional suffering, functional limitations, and diminished QoL. Patients with RA and SSc may have pain generators more frequently than patients without any underlying disorder. $\mathrm{NeP}$ is often associated with lower QoL and comorbid conditions such as neuropsychiatric disturbances. As QoL covers various aspects of physical and mental health, this has a major impact on the burden of disease. Our results show that RA patients with co-existing $\mathrm{NeP}$ had poorer physical and mental components of QoL, which may be associated with a heavier clinical burden of disease, resulting in significant direct and indirect costs for these patients and also for the health-care system.
Pain in SSc has been seldom evaluated [6]. Racine et al. [32] suggested that pain and itch have a detrimental impact in patients with SSc and are associated with poor status of mental and physical functions and HRQoL. Merz et al. [33] showed that emotional health, physical health, and social support are more relevant to pain than disease severity in SSc. Pain levels might remain stable with a small extent of improvement over time, and the severity of pain was shown to be associated with the type of disease.

Our study revealed statistically significant differences between $\mathrm{NeP}$-positive and $\mathrm{NeP}$-negative patients with RA in some outcome variables such as pain, fatigue, QoL, and psychological status. However, our results present cross-sectional data and did not include a healthy control population. Therefore, it is quite difficult to conclude whether these differences are clinically meaningful or not. Our study population was relatively small and had a female majority, which may be a limitation. A longitudinal follow-up of patients with RA and SSc regarding the changes in the NeP component and severity with time and treatments may reveal valuable data.

\section{Conclusion}

In conclusion, the existence of the $\mathrm{NeP}$ component is similar between patients with RA and patients SSc. However, $\mathrm{NeP}$ was associated with a heavier burden of disease in patients with RA.

\section{Statement of Ethics}

This study has been approved by the local ethics committee of our institution, and written informed consent according to the Declaration of Helsinki has been obtained from all patients.

\section{References}

1 Scott DL, Wolfe F, Huizinga TW. Rheumatoid arthritis. Lancet. 2010 Sep;376(9746): 1094-108.

2 Taylor P, Manger B, Alvaro-Gracia J, Johnstone R, Gomez-Reino J, Eberhardt E, et al. Patient perceptions concerning pain management in the treatment of rheumatoid arthritis. J Int Med Res. 2010 Jul-Aug;38(4):1213-24.

3 Medsger TA Jr. Natural history of systemic sclerosis and the assessment of disease activity, severity, functional status, and psychologic well-being [vi.]. Rheum Dis Clin North Am. 2003 May;29(2):255-73.
4 Gök K, Cengiz G, Erol K, et al. The Turkish Version of Multidimensional Assessment of Fatigue and Fatigue Severity Scale is reproducible and correlated with other outcome measures in patients with systemic sclerosis. Arch Rheumatol. 2016;31(4):329-32.

5 Karakulak UN, Okutucu S, Şahiner L, Maharjan N, Aladag E, Akdogan A, et al. Assessment of cardiac autonomic nervous system involvement in systemic sclerosis via exercise heart rate recovery. Med Princ Pract. 2015;24(1):17-22.
6 Georges C, Chassany O, Toledano C Mouthon L, Tiev K, Meyer O, et al. Impact of pain in health related quality of life of patients with systemic sclerosis. Rheumatology (Oxford). 2006 Oct;45(10):1298-302.

7 Schieir O, Thombs BD, Hudson M, Boivin JF, Steele R, Bernatsky S, et al.; Canadian Scleroderma Research Group. Prevalence, severity, and clinical correlates of pain in patients with systemic sclerosis. Arthritis Care Res (Hoboken). 2010 Mar;62(3):409-17.
Pain in Rheumatoid Arthritis and Systemic Sclerosis
Med Princ Pract 2018;27:537-542

DOI: $10.1159 / 000493480$ 
8 Bagnato G, Cordova F, Sciortino D, Miceli G, Bruno A, Ferrera A, et al. Association between cortisol levels and pain threshold in systemic sclerosis and major depression. Rheumatol Int. 2018 Mar;38(3):433-41.

9 Bagnato GL, Miceli G, Marino N, Sciortino D, Bagnato GF. Pulsed electromagnetic fields in knee osteoarthritis: a double blind, placebocontrolled, randomized clinical trial. Rheumatology (Oxford). 2016 Apr;55(4):755-62.

10 Lee YC, Lu B, Edwards RR, Wasan AD, Nassikas NJ, Clauw DJ, et al. The role of sleep problems in central pain processing in rheumatoid arthritis. Arthritis Rheum. 2013 Jan; 65(1):59-68.

11 Perrot S, Dieudé P, Pérocheau D, Allanore Y. Comparison of pain, pain burden, coping strategies, and attitudes between patients with systemic sclerosis and patients with rheumatoid arthritis: a cross-sectional study. Pain Med. 2013 Nov; 14(11):1776-85.

12 Koop SM, ten Klooster PM, Vonkeman HE Steunebrink LM, van de Laar MA. Neuropathic-like pain features and cross-sectional associations in rheumatoid arthritis. Arthritis Res Ther. 2015 Sep;17(1):237.

13 van Hecke O, Austin SK, Khan RA, Smith BH, Torrance N. Neuropathic pain in the general population: a systematic review of epidemiological studies. Pain. 2014 Apr;155(4):654-62.

14 Torrance N, Smith BH, Bennett MI, Lee AJ. The epidemiology of chronic pain of predominantly neuropathic origin. Results from a general population survey. J Pain. 2006 Apr; 7(4):281-9.

15 Joharatnam N, McWilliams DF, Wilson D, Wheeler M, Pande I, Walsh DA. A cross-sectional study of pain sensitivity, disease-activity assessment, mental health, and fibromyalgia status in rheumatoid arthritis. Arthritis Res Ther. 2015 Jan;17(1):11.

16 Lee YC, Cui J, Lu B, Frits ML, Iannaccone CK, Shadick NA, et al. Pain persists in DAS28 rheumatoid arthritis remission but not in ACR/EULAR remission: a longitudinal observational study. Arthritis Res Ther. 2011 Jun;13(3):R83.
17 Wolfe F, Boers M, Felson D, Michaud K, Wells GA. Remission in rheumatoid arthritis: physician and patient perspectives. J Rheumatol. 2009 May;36(5):930-3.

18 Geber C, Baumgärtner U, Schwab R, Müller $\mathrm{H}$, Stoeter P, Dieterich M, et al. Revised definition of neuropathic pain and its grading system: an open case series illustrating its use in clinical practice. Am J Med. 2009 Oct;122(10 Suppl):S3-12.

19 van den Hoogen F, Khanna D, Fransen J, Johnson SR, Baron M, Tyndall A, et al. 2013 classification criteria for systemic sclerosis: an American College of Rheumatology/European League against Rheumatism collaborative initiative. Arthritis Rheum. 2013 Nov;65(11): 2737-47.

20 Aletaha D, Neogi T, Silman AJ, Funovits J, Felson DT, Bingham CO 3rd, et al. 2010 rheumatoid arthritis classification criteria: an American College of Rheumatology/European League Against Rheumatism collaborative initiative. Ann Rheum Dis. 2010 Sep;69(9): $1580-8$.

21 Balke B. A Simple Field Test for the Assessment of Physical Fitness. Rep 63-6. Rep Civ Aeromed Res Inst US 1963:1-8.

22 Verkleij SP, Hoekstra T, Rozendaal RM, Waarsing JH, Koes BW, Luijsterburg PA, et al. Defining discriminative pain trajectories in hip osteoarthritis over a 2-year time period. Ann Rheum Dis. 2012 Sep;71(9):1517-23.

23 Bouhassira D, Attal N, Alchaar H, Boureau F, Brochet B, Bruxelle J, et al. Comparison of pain syndromes associated with nervous or somatic lesions and development of a new neuropathic pain diagnostic questionnaire (DN4). Pain. 2005 Mar;114(1-2):29-36.

24 Freynhagen R, Baron R, Gockel U, Tölle TR. painDETECT: a new screening questionnaire to identify neuropathic components in patients with back pain. Curr Med Res Opin. 2006 Oct;22(10):1911-20.

25 Celik S, Yenidunya G, Temel E, Purisa S, Uzum AK, Gul N, et al. Utility of DN4 questionnaire in assessment of neuropathic pain and its clinical correlations in Turkish patients with diabetes mellitus. Prim Care Diabetes. 2016 Aug;10(4):259-64.
26 Alkan H, Ardic F, Erdogan C, Sahin F, Sarsan A, Findikoglu G. Turkish version of the painDETECT questionnaire in the assessment of neuropathic pain: a validity and reliability study. Pain Med. 2013 Dec;14(12):1933-43.

27 Padua L, Briani C, Truini A, Aprile I, Bouhassirà $\mathrm{D}$, Cruccu $\mathrm{G}$, et al. Consistence and discrepancy of neuropathic pain screening tools DN4 and ID-Pain. Neurol Sci. 2013 Mar; 34(3):373-7.

28 Freynhagen R, Tölle TR, Gockel U, Baron R. The painDETECT project - far more than a screening tool on neuropathic pain. Curr Med Res Opin. 2016 Jun;32(6):1033-57.

29 Rifbjerg-Madsen S, Christensen AW, Boesen M, Christensen R, Danneskiold-Samsøe B, Bliddal H, et al. Can the painDETECT Questionnaire score and MRI help predict treatment outcome in rheumatoid arthritis: protocol for the Frederiksberg hospital's Rheumatoid Arthritis, pain assessment and Medical Evaluation (FRAME-cohort) study. BMJ Open. 2014 Nov;4(11):e006058.

30 Christensen AW, Rifbjerg-Madsen S, Christensen R, Dreyer L, Tillingsøe H, Seven S, et al. Non-nociceptive pain in rheumatoid arthritis is frequent and affects disease activity estimation: cross-sectional data from the FRAME study. Scand J Rheumatol. 2016 Nov; 45(6):461-9.

31 Ahmed S, Magan T, Vargas M, Harrison A, Sofat N. Use of the painDETECT tool in rheumatoid arthritis suggests neuropathic and sensitization components in pain reporting. J Pain Res. 2014 Oct; 7:579-88.

32 Racine M, Hudson M, Baron M, Nielson WR, Pope J, Markland J, et al.; Canadian Scleroderma Research Group. The Impact of Pain and Itch on Functioning and Health-Related Quality of Life in Systemic Sclerosis: An Exploratory Study. J Pain Symptom Manage. 2016 Jul;52(1):43-53.

33 Merz EL, Malcarne VL, Roesch SC, et al. Longitudinal patterns of pain in patients with diffuse and limited systemic sclerosis: integrating medical, psychological, and social characteristics. Qual Life Res. 2017 Jan;26(1):85-94. 